\title{
Ectopic Expression of an Arabidopsis Calmodulin-Like Domain Protein Kinase-Enhanced NADPH Oxidase Activity and Oxidative Burst in Tomato Protoplasts
}

\author{
Tim Xing, ${ }^{1}$ Xiao-Jing Wang, ${ }^{2}$ Kamal Malik, ${ }^{3}$ and Brian L. Miki ${ }^{3}$ \\ ${ }^{1}$ Agriculture and Agri-Food Canada, Cereal Research Centre, Winnipeg, Manitoba, Canada R3T 2M9; \\ ${ }^{2}$ Department of Biology, South China Normal University, Guangzhou, China 510631; ${ }^{3}$ Agriculture and \\ Agri-Food Canada, Eastern Cereal and Oilseed Research Centre, Ottawa, Ontario, Canada K1A 0C6 \\ Submitted 14 March 2001; Accepted 28 June 2001.
}

\begin{abstract}
Among plant defense responses to pathogen attack, the release of active oxygen species (AOS), termed the oxidative burst, may affect the attacking pathogen and the host plant cells at the infection site, thereby limiting the spread of the pathogen. Plasma membrane-associated NADPH oxidase represents a key enzyme in mediating the oxidative burst. The mechanisms of NADPH oxidase activation, however, remains unclear. Ectopic expression of AK1-6H, an Arabidopsis calmodulin-like domain protein kinase (CDPK) in tomato protoplasts enhanced plasma membrane-associated NADPH oxidase activity. Arabidopsis protein phosphatase $2 \mathrm{~A}$ abolished this enhancement, whereas Arabidopsis dual-specificity protein tyrosine phosphatase 1 or maize protein phosphatase 1 had no effect. tMEK2 ${ }^{\mathrm{MUT}}$, a constitutively activated, mitogen-activated protein kinase kinase from tomato, did not enhance NADPH oxidase activity when overexpressed. In a cell-free system, AK1-6H moderately stimulated the NADPH oxidase activity on plasma membrane. AK1-6H, but not tMEK2 ${ }^{\mathrm{MUT}}$, also enhanced production of $\mathrm{AOS}$ in intact protoplasts. Our results show that ectopic expression of a heterologous CDPK can enhance NADPH oxidase activity and stimulate an oxidative burst in tomato protoplasts.
\end{abstract}

Additional keywords: dephosphorylation, gene expression, phosphorylation.

Oxidative burst, the process of a striking release of active oxygen species by the host cells in response to pathogen attack, has been long studied in plants because it is one of the main rapid processes in plant defense mechanisms (Grant and Loake 2000; Lamb and Dixon 1997). One of the key enzymes in the oxidative burst is a plasma membrane-associated NADPH oxidase (Doke and Miura 1995; Mehdy 1994). In mammalian cells, this enzyme consists of two plasma membrane components ( $g p 91^{\text {phox }}$ and $p 22^{p h o x}$ ) and at least three cy-

Corresponding author: T. Xing; Telephone: +1-204-983-1465; Fax: +1-204-983-4604; E-mail: xingtx@em.agr.ca tosolic proteins ( $\mathrm{p} 47^{\text {phox }}$, $\mathrm{p} 67^{\text {phox }}$, and the monomeric $\mathrm{G}$ protein rac) (Jones 1994). It remains nonfunctional until cytosolic components dock on plasma membrane. Protein kinase $\mathrm{C}$ (PKC) plays a key role in phosphorylating cytosolic components of NADPH oxidase in neutrophil cells (Jones 1994). Studies based on kinase inhibitors, however, suggest that, in tomato, this role is probably filled by a $\mathrm{Ca}^{2+}$-dependent and calmodulin antagonist-sensitive protein kinase (Romeis et al. 2000; Xing et al. 1997).

Plant calmodulin-like domain protein kinases (CDPKs) share extensive sequence homology with the mammalian $\mathrm{Ca}^{2+}$ calmodulin $(\mathrm{CaM})$-dependent protein kinase II but contains a $\mathrm{CaM}$-like domain at the $\mathrm{C}$ terminus, which allows response to $\mathrm{Ca}^{2+}$ signals without CaM (Roberts and Harmon 1992). A constitutively activated Arabidopsis CDPK activated cold, dark, and osmotic stress response pathways (Sheen 1996). OsCDPK7, a rice CDPK, was induced by cold and high salinity (Saijo et al. 2000). Its overexpression in rice increased tolerance to cold and salt stress. Our previous study suggested that a tomato CDPK could be involved in the activation of oxidative burst (Xing et al. 1997), whereas another study suggested that a tomato CDPK is independent of or located upstream from a signaling pathway that is required for active oxygen species (AOS) production (Romeis et al. 2000). Genes encoding the CDPK(s) in the two studies, however, have not been identified or assigned. Arabidopsis AK1 represents one of the earliest CDPKs identified in plants, although its functional analysis is very limited. Because signaling elements isolated from one species may work in another species, as long as signaling interaction domains match between foreign proteins and endogenous signaling components (Xing and Jordan 2000), we investigated any possible functions of AK1 in the activation of NADPH oxidase activity and the production of AOS in tomato protoplasts.

Tomato plants (Lycopersicon esculentum cv. Bonny Best) were grown in peat soil in growth cabinets at $25^{\circ} \mathrm{C}$ under an illumination of $25 \mu \mathrm{E} \mathrm{m}^{-2} \mathrm{~s}^{-1}$ (16-h light/8-h dark). Protoplasts were prepared from fully expanded leaves (Xing et al. 2001) and kept on ice in W5 medium for $2 \mathrm{~h}$ prior to electroporation. Protoplasts were resuspended in electroporation buffer con- 
taining $150 \mathrm{mM} \mathrm{MgCl} 2$ and $0.4 \mathrm{M}$ mannitol at a density of $10^{6}$ protoplasts per $\mathrm{ml}$ and electroporated with 12 to $15 \mu \mathrm{g}$ of pMON999 carrying the AK1-6H gene (DDBJ/EMBL/GenBank accession no. L14771) under the $35 \mathrm{~S}$ promoter (Harper et al. 1994). Electroporation was performed at $200 \mathrm{~V}$ and $100 \mu \mathrm{F}$ (Gene Pulser II; Bio-Rad Laboratories, Hercules, CA, U.S.A.). Protoplasts were then allowed to recover on ice in the dark for $10 \mathrm{~min}$, followed by centrifugation at $500 \times g$ for $5 \mathrm{~min}$. Pellets were then supplemented with $500 \mu \mathrm{l}$ of incubation medium (Power and Chapman 1985) and incubated in the dark at $30^{\circ} \mathrm{C}$ for $24 \mathrm{~h}$. Isolation and overexpression in protoplasts of other genes, including tomato tMEK2 $2^{\mathrm{MUT}}$ (Xing et al. 2001), Arabidopsis dual-specificity protein tyrosine phosphatase (DsPTP1) (DDBJ/EMBL/GenBank accession no. Y18620), maize protein phosphatase 1 (PP1) (DDBJ/EMBL/GenBank accession no. M60215), and Arabidopsis protein phosphatase 2A (PP2A) (DDBJ/EMBL/GenBank accession no. U39568), were described previously (Xing et al. 2001).

Following overexpression of AK1-6H, protoplasts were pooled and plasma membranes were isolated (Xing et al. 1996). Production of $\mathrm{O}_{2}^{-}$by NADPH oxidase was monitored at $25^{\circ} \mathrm{C}$ following the superoxide dismutase-inhibitable and NADPH-dependent reduction of cytochrome $c$ at $550 \mathrm{~nm}$ as described previously (Xing et al. 1997). AK1-6H significantly increased NADPH oxidase activity, whereas tMEK2 $2^{\mathrm{MUT}}$, which was shown to activate an array of pathogenesis-related genes in the same protoplast system (Xing et al. 2001), did not have any effect (Fig. 1). We expressed AK1-6H only in its fusion form. A previous study indicates that a purified AK1-6H fusion protein displayed kinase activity comparable to a native CDPK (Harper et al. 1994). Additionally, eight different protein kinases including CDPKs were fused to double hemagglutinin epitope tag at the $\mathrm{C}$ terminus for expression in maize protoplasts in stress studies, and the fusion proteins were suggested to reflect the activities of native wild-type protein kinases (Sheen 1996).

Phosphorylation and dephosphorylation is an effective mechanism for posttranslational modification of proteins. We ex-

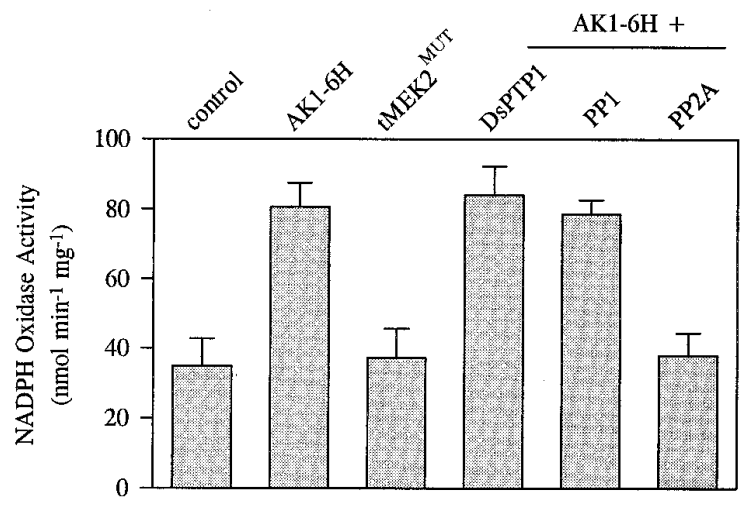

Fig. 1. Effect of ectopic expression of protein kinases and phosphatases on plasma membrane NADPH oxidase activity. Plasma membraneassociated NADPH oxidase activity was measured following overexpression of the AK1-6H or tMEK2 ${ }^{\mathrm{MUT}}$ gene or following coexpression of AK1-6H and dual-specificity protein tyrosine phosphatase (DsPTP1), protein phosphatase 1 (PP1), or protein phosphatase 2A (PP2A). Values for NADPH oxidase activity are the mean \pm standard error $(n=4)$. NADPH oxidase activity for plasma membrane isolated from control protoplasts was $34.82 \mathrm{nmol} / \mathrm{min} / \mathrm{mg}$ of protein. amined the effect of different protein phosphatases on the activation of NADPH oxidase by AK1-6H. When AK1-6H was co-expressed in protoplasts with the Arabidopsis DsPTP1 or maize PP1, the activation of NADPH oxidase was not affected significantly, but overexpression of Arabidopsis PP2A significantly inhibited AK1-6H-induced activation of NADPH oxidase (Fig. 1).

Reconstruction of a cell-free system, which consists of isolated plasma membrane and cytosol, is an effective way to study the activation mechanisms of NADPH oxidase. Unlike neutrophil cells, however, a cell-free system could not be reconstructed simply by using isolated plasma membranes and isolated cytosols from untreated plant cells with Arabidopsis (Desikan et al. 1996) or tomato (Xing et al. 1997). The production of superoxide was initiated by the reconstruction of Arabidopsis plasma membrane with neutrophil cytosolic fractions (Desikan et al. 1996). Recombinant AK1-6H that was sandwiched between a glutathione S-transferase and $6 \times$ histidine tag was expressed in Escherichia coli (XL-1 blue), and the expressed fusion protein was collected by affinity chromatography. Plasma membranes from tomato leaves were isolated (Xing et al. 1996) and suspended in activation buffer $(118 \mathrm{mM} \mathrm{NaCl} ; 4.7 \mathrm{mM} \mathrm{KCl} ; 25 \mathrm{mM} \mathrm{Na}-\mathrm{N}$-2-hydroxyethylpiperazine- $N^{\prime}$-2-ethanesulfonic acid; $1.2 \mathrm{mM} \mathrm{KH}_{2} \mathrm{PO}_{4}$; $1.2 \mathrm{mM} \mathrm{MgSO}$; $0.1 \mathrm{mM} \mathrm{CaCl}_{2}$; and $5.5 \mathrm{mM}$ glucose, $\mathrm{pH}$ 7.4). When purified AK1-6H was incubated with plasma membranes, NADPH oxidase activity was increased (Fig. 2). Withdrawal of $\mathrm{Ca}^{2+}$ from the reaction mixture completely abolished the effect of AK1-6H (Fig. 2). Increasing the dosage of AK1$6 \mathrm{H}$ did not result in a higher recovery of NADPH oxidase activity (data not shown). When recombinant tMEK2 ${ }^{\text {MUT }}$ was added to plasma membranes, it did not have any effect (Fig. 2 ). This could be explained as a result of the observation that plasma membrane may not contain the required components of MAP kinase pathways (Hirt 1997). Control of NADPH oxidase activity involves a phosphorylation and dephosphorylation system on plasma membrane, and the background activity of NADPH oxidase is maintained on plasma membrane

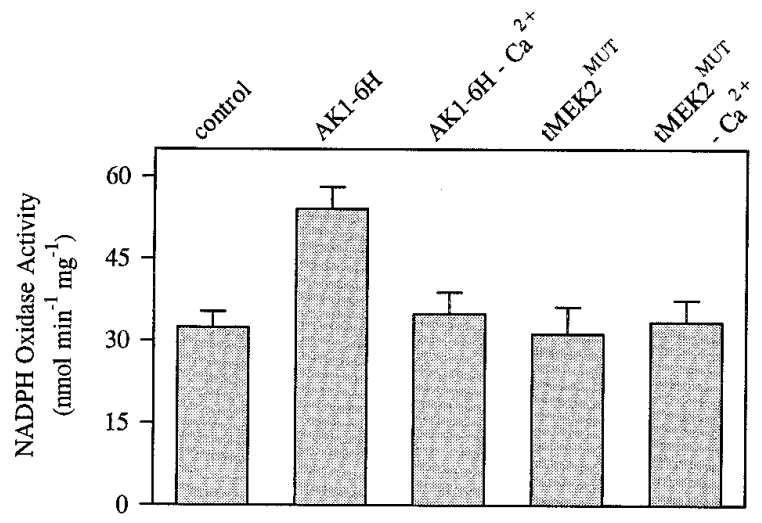

Fig. 2. Effect of AK1-6H and tMEK2 $2^{\mathrm{MUT}}$ enzymes on NADPH oxidase activity in a cell-free system. Plasma membrane was isolated from tomato leaves and $20 \mu \mathrm{g}$ was incubated with AK1-6H (200 ng) or tMEK2 ${ }^{\mathrm{MUT}}\left(500 \mathrm{ng}\right.$ ) at $30^{\circ} \mathrm{C}$ for $6 \mathrm{~min}$ in the presence or absence of $\mathrm{Ca}^{2+}(0.1 \mathrm{mM})$. NADPH oxidase activity was then measured. Values for NADPH oxidase activity are the mean \pm standard error $(n=4)$. NADPH oxidase activity for plasma membrane isolated from control protoplasts was $32.35 \mathrm{nmol} / \mathrm{min} / \mathrm{mg}$ of protein. 
through a combined effect of kinases and phosphatases (Xing et al. 1997). The addition of AK1-6H to the cell-free system may have increased phosphorylation of the appropriate components on the plasma membrane and hence activated the enzyme, although only to a limited level. A purified phospholipiddependent rat brain PKC (CalBiochem, San Diego, CA, U.S.A.) was tested in the cell-free system but failed to affect NADPH oxidase activity (data not shown).

Because a cell-free system does not completely reflect the mechanisms in intact cells, we further examined the effect of AK1-6H on the generation of AOS in intact protoplasts. We adopted a method that has been used successfully to detect AOS in tomato leaves and cell suspension cultures (Higgins et al. 1998; Lu and Higgins 1998) with fluorescent probe $2^{\prime}, 7^{\prime}$ dichlorofluorescein diacetate (DCFH-DA) that, after deacetylation in the cell, reacts with $\mathrm{H}_{2} \mathrm{O}_{2}$ in the presence of peroxidase to form fluorescent product $2^{\prime}, 7^{\prime}$-dichlorofluorescein (DCF). Following transient expression of AK1-6H or tMEK2 $2^{\mathrm{MUT}}$, DCFH-DA was added to the protoplast suspension. DCF in protoplasts was visualized under a D-7082 Universal fluorescent microscope (Zeiss, Oberkochen, Germany). $\mathrm{H}_{2} \mathrm{O}_{2}$ accumulation was increased significantly in protoplasts transformed with AK1-6H (Fig. 3). Overexpression of tMEK2 ${ }^{\mathrm{MUT}}$ did not increase DCF production. It has been suggested that the production of AOS is probably independent of MAP kinase pathways (MAPK) (Romeis et al. 1999), although a recent study suggests that the hypersensitive response could be activated by the MAPK pathway (Yang et al. 2001). Probably the most interesting observation is that a signaling network of oxidative burst and cognate redox in Arabidopsis functions independently of ethylene, salicylic acid, and methyl jasmonate but is dependent on MAP kinase kinase (MAPKK) activity (Grant et al. 2000). The bifurcating nature of this redox signaling pathway that possesses a MAPK module has been suggested to contribute to the establishment of plant disease resistance and to the development of cellular protectant mechanisms (Grant et al. 2000). Additional complexity also is associated with the relationship of oxidative burst and hypersensitive cell death. Yano et al. (1999) examined the role of AOS in hypersensitive cell death in tobacco cells, which is induced by xylanase from Trichoderma viride, and the results suggest that there is an oxidative burst-independent pathway that leads to hypersensitive cell death.

Defense mechanism studies in plants have benefited greatly from mammalian and yeast models. Although some signal transduction pathways are common, many pathways, pathway components, or functions of those components are unique to plants (Arabidopsis Genome Initiative 2000; Xing and Jordan 2000). The key defense pathway leading to AOS production differs between plants and mammals. In the mammalian pathway, the assembly and activation of NADPH oxidase requires PKC (Jones 1994). Tomato cells, however, use a CDPK in this process when a defense response is triggered in an incompatible interaction between tomato and fungal pathogen Cladosporium fulvum (Xing et al. 1997). A possible direct involvement of $G$ proteins in the activation of a $\mathrm{Ca}^{2+}$ channel and a CDPK has been suggested (Higgins et al. 1998) on the basis of the action of $\mathrm{G}$ protein inhibitors and activators on elicitor activation of $\mathrm{Ca}^{2+}$ channels (Gelli et al. 1997) as well as the absence of a requirement for diacylglycerol, which is necessary to activate PKC during NADPH oxidase assembling in neutrophil cells.
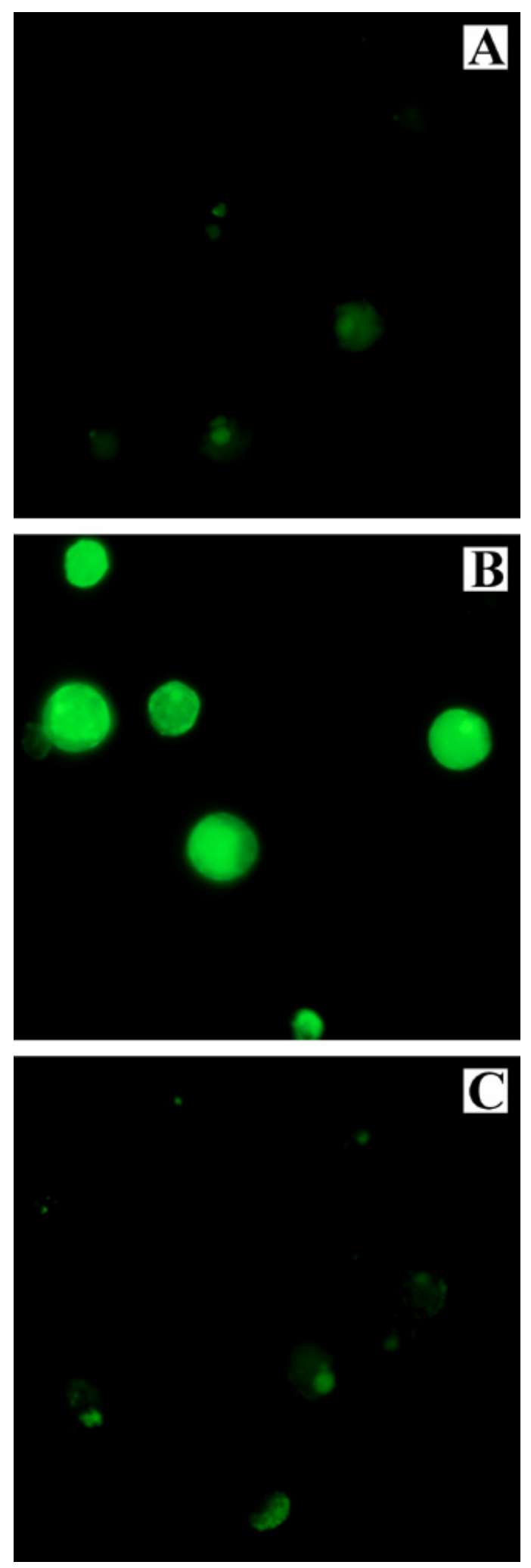

Fig. 3. Detection of active oxygen species production in intact tomato protoplasts. Following transient expression of AK1-6H or tMEK2 ${ }^{\mathrm{MUT}}$, $2^{\prime}, 7^{\prime}$-dichlorofluorescein diacetate was added to the protoplast suspension. The $2^{\prime}, 7^{\prime}$-dichlorofluorescein in protoplasts was visualized under a fluorescent microscope. A, Control. B, Protoplasts transformed with AK1-6H. C, Protoplasts transformed with tMEK2 ${ }^{\mathrm{MUT}}$. 
Sophisticated mechanisms of CDPK involvement in the activation of NADPH oxidase, however, is complicated. Cloned plant NADPH oxidases possess a N-terminal extension with two functional $\mathrm{Ca}^{2+}$-binding motifs (Keller et al. 1998; Torres et al. 1998). Our study suggests a possible role for CDPK in the control of NADPH oxidase activity and AOS production in tomato cells.

\section{ACKNOWLEDGMENTS}

This work was supported by a research grant from Agriculture and Agri-Food Canada Genomics Initiative to T. Xing and a Matching Investment Initiative grant to B. L. Miki. We thank J. Harper of The Scripps Research Institute (U.S.A.) for the gift of the AK1-6H clone and T. Martin for technical help. We gratefully acknowledge H. Hirt of the University of Vienna and J. Sheen of Massachusetts General Hospital (U.S.A.) for helpful discussions, and B. McCallum and C. Rampitsch of Agriculture and Agri-Food Canada for critically reading the manuscript. This is ECORC publication 001606.

\section{LITERATURE CITED}

Arabidopsis Genome Initiative. 2000. Analysis of the genome sequence of the flowering plant Arabidopsis thaliana. Nature 408:796-815.

Desikan, R., Hancock, J. T., Coffey, M. J., and Neill, S. J. 1996. Generation of active oxygen in elicited cells of Arabidopsis thaliana is mediated by a NADPH oxidase-like enzyme. FEBS (Fed. Eur. Biochem. Soc.) Lett. 382:213-217.

Doke, N., and Miura, Y. 1995. In vitro activation of NADPH-dependent $\mathrm{O}_{2}$-generating system in a plasma membrane-rich fraction of potato tuber tissues by treatment with an elicitor from Phytophthora infestans or with digitonin. Physiol. Mol. Plant Pathol. 46:17-28.

Gelli, A., Higgins, V. J., and Blumwald, E. 1997. Activation of plant plasma membrane $\mathrm{Ca}^{2+}$-permeable channels by race-specific fungal elicitors. Plant Physiol. 113:269-279.

Grant, J. J., and Loake, G. J. 2000. Role of reactive oxygen intermediates and cognate redox signaling in disease resistance. Plant Physiol. 124:21-29.

Grant, J. J., Yun, B. W., and Loake, G. J. 2000. Oxidative burst and cognate redox signalling reported by luciferase imaging: Identification of a signal network that functions independently of ethylene, SA, and Me-JA but is dependent on MAPKK activity. Plant J. 24:569-582.

Harper, J. F., Huang, J.-F., and Lloyd, S. J. 1994. Genetic identification of an autoinhibitor in CDPK, a protein kinase with a calmodulin-like domain. Biochemistry 33:7267-7277.

Higgins, V. J., Lu, H., Xing, T., Gelli, A., and Blumwald, E. 1998. The gene-for-gene concept and beyond: Interactions and signals. Can. J. Plant Pathol. 20:150-157.

Hirt, H. 1997. Multiple roles of MAP kinases in plant signal transduction. Trends Plant Sci. 2:11-15.

Jones, O. T. G. 1994. The regulation of superoxide production by the NADPH oxidase of neutrophils and other mammalian cells. Bio-
Essays 16:919-923

Keller, T., Damude, H. G., Werner, D., Doerner, P., Dixon, R. A., and Lamb, C. 1998. A plant homolog of the neutrophil NADPH oxidase gp91 ${ }^{\text {phox }}$ subunit gene encodes a plasma membrane protein with $\mathrm{Ca}^{2+}$ binding motifs. Plant Cell 10:255-266.

Lamb, C., and Dixon, R. A. 1997. The oxidative burst in plant disease resistance. Annu. Rev. Plant Physiol. Plant Mol. Biol. 48:251-275.

Lu, H., and Higgins, V. J. 1998. Measurement of active oxygen species generated in planta in response to elicitor AVR9 of Cladosporium fulvum. Physiol. Mol. Plant Pathol. 52:35-51.

Mehdy, M. C. 1994. Active oxygen species in plant defense against pathogens. Plant Physiol. 105:467-472.

Power, J. B., and Chapman, J. V. 1985. Isolation, culture and genetic manipulation of plant protoplasts. Pages 113-125 in: Plant Cell Culture: A Practical Approach. E. A. Dixon, ed. IRL Press, Oxford.

Roberts, D. M., and Harmon, A. C. 1992. Calcium-modulated proteins: Targets of intracellular calcium signals in higher plants. Annu. Rev. Plant Physiol. Plant Mol. Biol. 43:375-414.

Romeis, T., Piedras, P., Zhang, S., Klessig, D. F., Hirt, H., and Jones, J. D. G. 1999. Rapid Avr9- and Cf-9-dependent activation of MAP kinases in tobacco cell cultures and leaves: Convergence of resistance gene, elicitor, wound, and salicylate responses. Plant Cell 11:273-287.

Romeis, T., Piedras, P., and Jones, J. D. G. 2000. Resistance gene-dependent activation of a calcium-dependent protein kinase in the plant defense response. Plant Cell 12:803-815.

Saijo, Y., Kinoshita, N., Hata, S., Kyozuka, J., Shimamoto, K., and Izui, K. 2000. Overexpression of a single calcium-dependent protein kinase confers both cold- and salt-drought tolerance on rice plants. Plant J. 23:319-328.

Sheen, J. 1996. $\mathrm{Ca}^{2+}$-dependent protein kinase and stress signal transduction. Science 274:1900-1902.

Torres, M. A., Onouchi, H., Hamada, S., Machida, C., HammondKosack, K. E., and Jones, J. D. G. 1998. Six Arabidopsis thaliana homologues of the human respiratory burst oxidase $\left(g p 91^{\text {phox }}\right)$. Plant J. 14:365-370.

Xing, T., and Jordan, M. 2000. Genetic engineering of plant signal transduction mechanisms. Plant Mol. Biol. Rep. 18:309-318.

Xing, T., Higgins, V. J., and Blumwald, E. 1996. Regulation of plant defense response to fungal pathogens: Two types of protein kinases in the reversible phosphorylation of the host plasma membranes $\mathrm{H}^{+}$ATPase. Plant Cell 8:555-564.

Xing, T., Higgins, V. J., and Blumwald, E. 1997. Race-specific elicitors of Cladosporium fulvum promote translocation of cytosolic components of NADPH oxidase to the plasma membrane of tomato cells. Plant Cell 9:249-259.

Xing, T., Malik, K., Martin, T., and Miki, B. L. 2001. Activation of tomato $\mathrm{PR}$ and wound-related genes by a mutagenized tomato MAP kinase kinase through divergent pathways. Plant Mol. Biol. 46:109-120.

Yang, K. Y., Liu, Y. D., and Zhang, S. Q. 2001. Activation of a mitogenactivated protein kinase pathway is involved in disease resistance in tobacco. Proc. Natl. Acad. Sci. U.S.A. 98:741-746.

Yano, A., Suzuki, K., and Shinshi, H. 1999. A signaling pathway, independent of the oxidative burst, that leads to hypersensitive cell death in cultured tobacco cells includes a serine protease. Plant J. 18: 105-109. 